\title{
Damped Zigzag Upward Motion of a Ball in Fluid: a Numerical Model
}

\author{
Muhammad Nur Tajuddin ${ }^{a, b}$, Novitrian $^{c}$, Euis Sustini ${ }^{d}$, Veinardi Suendo ${ }^{e}$, and Sparisoma Viridi, \\ ${ }^{a}$ SMA Negeri 2 Palu, Palu, Sulawesi Tengah \\ ${ }^{b}$ Master Program in Physics Teaching, \\ FMIPA, Institut Teknologi Bandung, Bandung \\ ${ }^{c}$ Nuclear Physics and Biophysics Research Division, \\ FMIPA, Institut Teknologi Bandung, Indonesia \\ ${ }^{d}$ Physics of Electronic Materials Research Division, \\ FMIPA, Institut Teknologi Bandung, Indonesia \\ ${ }^{e}$ Inorganic and Physical Chemistry Research Division, \\ FMIPA, Institut Teknologi Bandung, Indonesia \\ e-maildudung@fi.itb.ac.id
}

\begin{abstract}
Several types of upward motion of a ball in a cylinder filled with water are observed in experiment such as straight, damped zigzag, and zigzag upward motion. Center of mass of the balls and the way the balls are released play important role to determine the type of occuring upward motion. A simple theoretical model regarding only gravitational force, Stokes drag force, and Archimedes bouyancy force, where the work points of these forces are not coincident, are developed and used to explain the damped zigzag motion.
\end{abstract}

Keywords: Damped zigzag motion, Viscosity, Falling ball, Stokes force, Archimedes force

\section{Introduction}

The falling ball viscometer is one of the most well known simple apparatus to measure fluid viscosity $^{1)}$. Simplicity of the model proposed theoretically by Stokes makes this model easy to be calculated and implemented, There is an assumption that the Stokes drag force can work in any direction of ball motion, not only in downward direction as it was derived for laminar flow moving againts the ball ${ }^{2}$. Unfortunately using only Stokes drag force can not explain several types of motion observed, such as rotating, spinning, fluctuating, and straight upward motion $^{3}$. In this work a simple model based on Stokes model are proposed and calculated. The results are then compared with experimental results.

\section{Theoretical model}

2.1 Straight upward motion (linear motion)

The gravitational force $\vec{F}_{G}$ in downward direction, Archimedes buoyant force $\vec{F}_{A}$ in upward direction, and Stokes drag force $\vec{F}_{S}$ in opposite direction of the motion are the only forces considered in the model. Each force works only at certain point labeled like its name, for example $\vec{F}_{G}$ works only at point $G$. In general, point $S, A$, and $G$ are not always at the same position. In the special case as shown in Figure 1 the ball can move upward in a straight line because these three points sit in a straight vertical line. This configuration introduces no torque, so that the ball will move linearly.

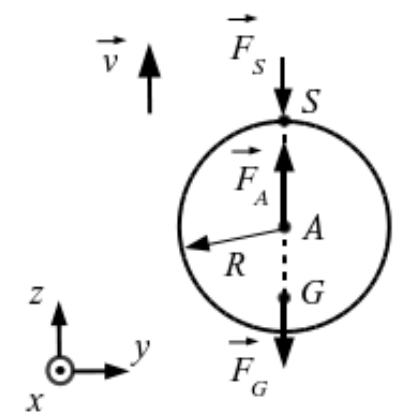

Figure 1. A ball with radius $R$ moving upward under influence of gravitational force $\vec{F}_{G}$, Archimedes bouyant force $\vec{F}_{A}$, and Stokes drag force $\vec{F}_{S}$.

Gravitational force $\vec{F}_{G}$ is expressed in term of ball density $\rho_{b}$, earth gravitational acceleration $g$, and ball volume $V$,

$$
\vec{F}_{G}=-\rho_{b} g V \hat{k} .
$$

In the same way also for expressing Archimedes bouyant force $\vec{F}_{A}$

$$
\vec{F}_{A}=\rho_{f} g V \hat{k},
$$

where $\rho_{f}$ is fluid density. According to Stokes, moving ball in a fluid must face resistance of the fluid proportional to and against direction of velocity $\vec{v}$ in term of Stokes drag force $\vec{F}_{S}$

$$
\vec{F}_{S}=-6 \pi \eta R \vec{v},
$$

where $\eta$ is viscosity of the fluid and $R$ is the ball radius. Combining Equations (1) - (3) using Newton's 
second law of linear motion, an expression of ball acceleration $\vec{a}$ can be found

$$
\vec{a}=\frac{\left(\rho_{f}-\rho_{b}\right) g V \hat{k}-6 \pi \eta R \vec{v}}{m} .
$$

The ball terminal velocity $\vec{v}_{T}$ can be found by setting $\vec{a}=0$ in Equation (4), which gives

$$
\vec{v}_{T}=\frac{2\left(\rho_{f}-\rho_{b}\right) g R^{2}}{9 \eta} \hat{k} .
$$

\subsection{Pendulum-like motion (rotational motion)}

When the three forces considered in this model do not work as shown in Figure 1 they will introduce a torque to the system. Gravitational force $\vec{F}_{G}$ at point $G$ always points downward and Archimedes bouyant force $\vec{F}_{A}$ at point $A$ always points upward.

It is proper to the take ball's center of mass at point $G$ as the rotation point. But since the goal is to obtain a zigzag type motion, it is more useful to take point $S$ as center of rotation. This point is not always the same because it changes as face of the ball rotates.

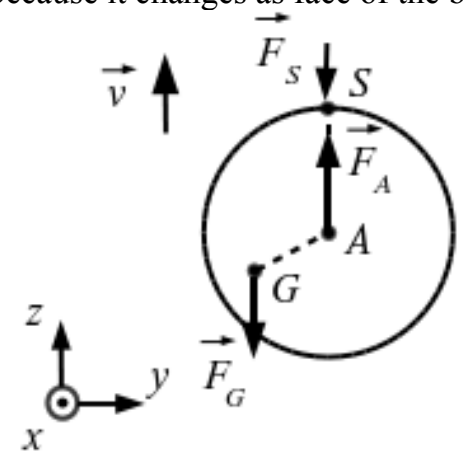

Figure 2. As the ball moves to direction of $\vec{v}$, gravitational force $\vec{F}_{G}$ always points downward and Archimedes bouyancy force $\vec{F}_{A}$ always points upward, where Stokes drag force $\vec{F}_{S}$ always againts the direction of motion.

First, the emerging torque at point $S$ in this configuration must be also taken into account, which are

$$
\vec{\tau}_{S}=\vec{r}_{G S} \times \vec{F}_{G}
$$

torque caused by gravitational force, where $\vec{r}_{G S}=\vec{r}_{G}-\vec{r}_{S}$, position of point $G$ measured from point $S$. Since upward motion is dominant, it is assumed that point $S$ and $A$ are always located in vertical line. Then, using Newton second law of angular motion

$$
\vec{\alpha}_{S}=\frac{\vec{\tau}_{S}}{I}
$$

angular acceleration $\vec{\alpha}_{S}$ at point $S$ can be found, where $I$ is ball's momen inertia. In this case moment of inertia of the ball is

$$
I=\frac{2}{5} m R^{2}+m R^{2},
$$

which is derived using paralel-axis theorem [4].

\subsection{Combination of linear and rotational motion}

Zigzag upward motion then is just as simple as a superposition of motion of the straight upward linear motion and the rotational motion. There are also initial conditions such as initial angular velocity, initial linear velocity, initial position, and initial angular position (or orientation), which must be considered. For example

$$
\begin{aligned}
& \vec{r}(0)=0 \\
& \vec{v}(0)=0 \\
& \bar{\theta}(0)=0 \\
& \vec{\omega}(0)=0
\end{aligned}
$$

can be used as initial condition.

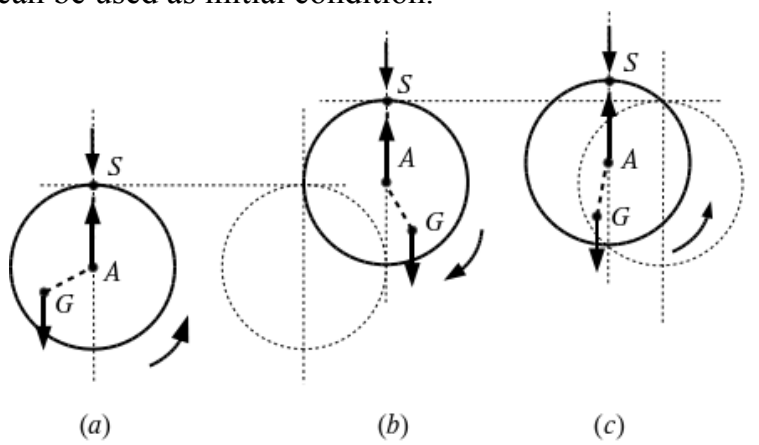

Figure 3. A pendulum-like motion combined with upward straight motion will produce a damped zigzag motion as observed in experiments.

It is illustrated in Figure 3 how an oscillating pendulum-like motion combined with upward straight motion can produce the desired zigzag, damped oscillating motion. Explanations are as follows: $(a)$ as the ball moves in upward direction indicated against the arrow at point $S$, the force at $G$ produces torque at $S$, which then rotates the ball in counter clockwise direction; (b) Then point $S$ is changed, it must be always in front of the ball. Previous process reoccurres, but this time the ball rotates in clockwise direction; and (c) The same type of motion as in (a) is repeated but with smaller amplitude.

\section{Calculation Method}

Euler methods ${ }^{5)}$ are used to solve the differential equations concerning Equation (4) for linear motion and Equation (6) - (9) for rotational motion. Numeric equations are listed below

$$
\begin{aligned}
& \vec{F}_{A}^{(i)}=\rho_{f} g V \hat{k}, \\
& \vec{F}_{G}^{(i)}=-\rho_{b} g V \hat{k} . \\
& \vec{F}_{S}^{(i)}=-6 \pi \eta R \vec{v},
\end{aligned}
$$




$$
\begin{aligned}
& \vec{r}_{G S}^{(i+1)}=\vec{r}_{A}^{(i)}+\left|\vec{r}_{G S}^{(i+1)}\right|\left(\hat{j} \sin \theta^{(i)}-\hat{k} \cos \theta^{(i)}\right), \\
& \vec{\alpha}_{S}^{(i)}=\frac{5 \vec{r}_{G S}^{(i)} \times \vec{F}_{G}^{(i)}}{7 m R^{2}}, \\
& \vec{\omega}_{S}^{(i+1)}=\vec{\omega}_{S}^{(i)}+\vec{\alpha}_{S}^{(i)} \Delta t, \\
& \vec{\theta}_{S}^{(i+1)}=\vec{\theta}_{S}^{(i)}+\vec{\omega}_{S}^{(i)} \Delta t+\frac{1}{2} \vec{\alpha}_{S}^{(i)}(\Delta t)^{2}, \\
& \vec{a}_{A}^{(i)}=\frac{\vec{F}_{A}^{(i)}+\vec{F}_{G}^{(i)}+\vec{F}_{S}^{(i)}}{m}, \\
& \vec{v}_{A}^{(i+1)}=\vec{v}_{A}^{(i)}+\vec{a}_{A}^{(i)} \Delta t, \\
& \vec{r}_{A}^{(i+1)}=\vec{r}_{A}^{(i)}+\vec{v}_{A}^{(i)} \Delta t+\frac{1}{2} \vec{a}_{A}^{(i)}(\Delta t)^{2}, \\
& t^{(t+1)}=t^{(t)}+\Delta t .
\end{aligned}
$$

Using iteration by increasing $i$ trajectory of ball motion can be solved and plotted.

\section{Results and Discussion}

\subsection{Straight upward motion (linear motion)}

By using the set of numerical equations showed in Equation (12) - (22) with intial condition: $R=1.2 \mathrm{~cm}, \eta=1 \mathrm{cPs}, \rho_{b}=0.95 \mathrm{~g} / \mathrm{cm}^{3}, \rho_{f}=1.00$ $\mathrm{g} / \mathrm{cm}^{3}, \Delta t=1 \times 10^{-5} \mathrm{~s}, r_{A G}=2 \mathrm{~mm}$, and $g=9.81$ $\mathrm{m} / \mathrm{s}^{2}$, value of terminal velocity $v_{T}$ as shown in Equation (5) is obtained, which is $1.5696 \times 10^{-5} \mathrm{~m} / \mathrm{s}$. Velocity time series is plotted in Figure 4 . This result ensures that the algorithms valid for simple upward linear motion. As shown in Figure 4 value of terminal velocity $v_{T}$ is reach in about $2 \times 10^{-4} \mathrm{~S}$ as the ball is released from its initial position.

Since in our experiment we can not measure such a small time in order of $10^{-5} \mathrm{~m} / \mathrm{s}$, this result is not yet confirmed.

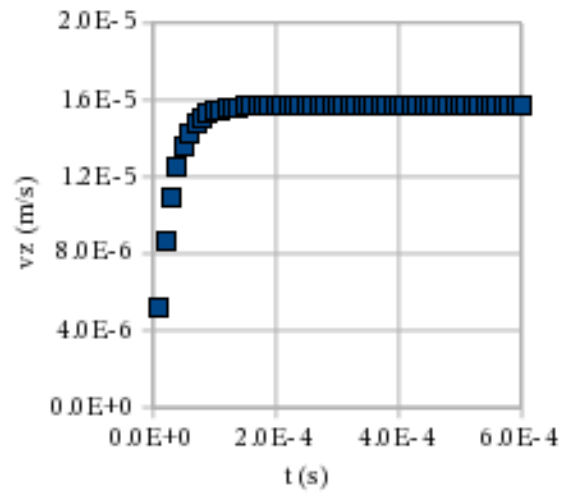

Figure 4. Ball vertical velocity $v_{z}$ time series for liniear upward motion, where terminal velocity obtained $v_{T}=1.5696 \times 10^{-5} \mathrm{~m} / \mathrm{s}$ is exactly the same as predicted in Equation (5).

\subsection{Pendulum-like motion (rotational motion)}

Using the same parameters as previous in the previous result with additon parameter: $\theta_{0}=0.157 \mathrm{rad}$ or $9^{\circ}$ we get result of angle of swinging center of mass or point $G$ with rotation point at point $S$ as shown previously in Figure 3.

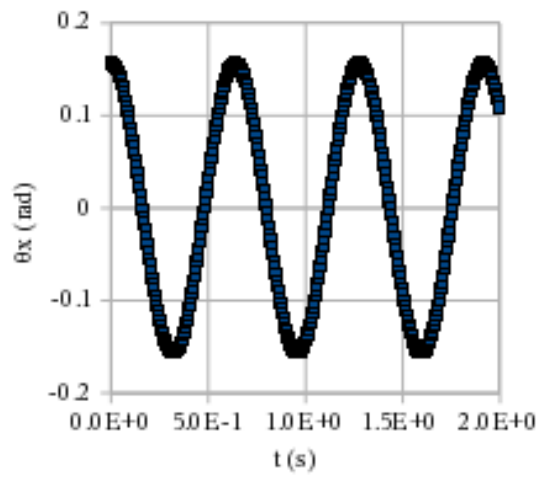

Figure 5. Time series of angle of center of mass to vertical direction $\theta_{x}$, where periode $T$ has value of 0.64 $\mathrm{s}$ as predicted by Equation (23).

According to the theory in [4] for paralel-axes theorem in calculating momen inertia $I$ at point $S$ and in [6] for periode of a physical pendulum in form of

$$
T=2 \pi \sqrt{\frac{I}{m g L}},
$$

where $L$ is the same as our $r_{G S}$ in this case, we get value of $T=0.64 \mathrm{~s}$ as shown in Figure 5 .

\subsection{Combination of linear and rotational motion}

Now both motion in section 4.1 and 4.2 will be combined to get the desired damped zigzag motion through a relation in form of

$$
\vec{v}_{A}^{(i+1)}=\vec{v}_{A}^{(i)}+\vec{r}_{G S} \times \vec{\omega}_{S} .
$$

As a result it can be seen in Figure 6 a zigzag motion of the ball. As a comparison is series of experiment photos illustrated in Figure 7.

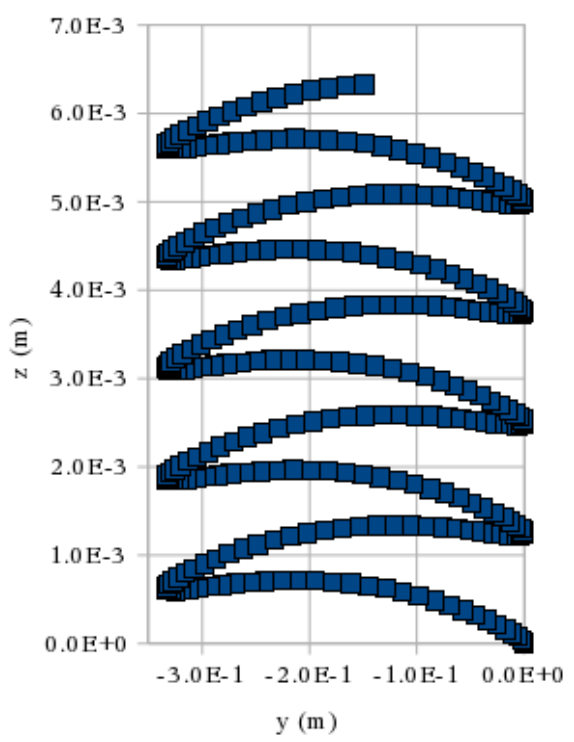

Figure 6. Simulation resuls for zigzag motion with the parameters as desribed in text. 


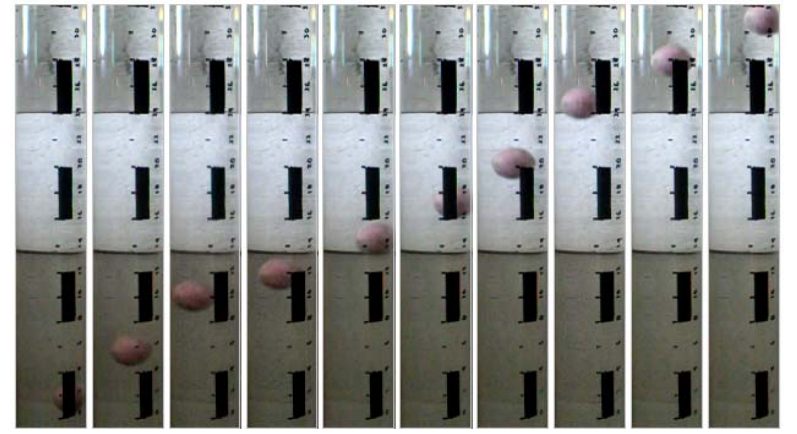

Figure 7. Example of experiment results: time is increasing from left to right. Periodic black rectangles are marks for measuring ball position every $4 \mathrm{~cm}$.

Ball rises up with a zigzag motion as it can be seen in Figure 7. It moves to the left and to right periodically at the same time as it moves upward. It oscillates in periode about $11.2 \mathrm{~s}$ with some length about $17 \mathrm{~cm}$.

\section{Conclusion}

In this work we can conclude that our model shows that the zigzag motion is a superposition of an upward straight motion and a pendulum-like motion.

\section{Acknowledgement}

Author would like to thank the scholarship which is made available in frame of cooperation (KERMA) between Departemen Pendidikan Nasional Indonesia (Diknas) and Institut Teknologi Bandung
(ITB) in year 2007-2008. Calculation part of this work is supported by Institut Teknologi Bandung Alumni Association (IA-ITB) under Research Grant No. $1244 \mathrm{a} / \mathrm{K} 01.7 / \mathrm{PL} / 2008$. We would like to thank A. Rusli for his comprehensif reading and his intensive comments.

\section{References}

1. Hou Yu, Fan Jie, and Liu Jinyan, A Research About the Viscosity Measurement Technology, http://www.paper.edu.cn/, 10-30(2008)

2. G. G. Stokes, Proc. Cambridge Philos. Soc. 9, 8 1851.

3. M. Nur Tajuddin and S. Viridi, Determination of Fluids Viscosity using Bouyancy of a Moving Ball in a Pipe with Different Angels, Proceeding of International Conference of Mathematics and Natural Sciences, Bandung, 28-30 Oktober 2008, ED-P-3, 8 pages.

4. D. Halliday, R. Resnick, and J. Walker, Fundamentals of Physics, John Wiley \& Sons, Ext. Eighth Edition, 253-254, 2008.

5. N. Gershenfeld, The Nature of Mathematical Modeling, Cambridge University Press, Third reprint of first edition, 67-70, 2002.

6. P. M. Fisbane, S. Gasiorowicz, and S. T. Thornton, Physics for Scientists and Engineers, Prentices Hall, New Jersey, Extended, Second Edition, 369-360, 1996. 